\title{
Foncier, pouvoirs locaux et décentralisation dans le département de Dakoro (Niger)
}

\section{Abdoulaye Mohamadou}

\section{(e) OpenEdition \\ 1 Journals}

Édition électronique

URL : http://journals.openedition.org/apad/4052

DOI : 10.4000/apad.4052

ISSN : 1950-6929

Éditeur

LIT Verlag

\section{Édition imprimée}

Date de publication : 1 décembre 2010

Pagination : 105-135

ISBN : 978-3-643-10535-6

\section{Référence électronique}

Abdoulaye Mohamadou, «Foncier, pouvoirs locaux et décentralisation dans le département de Dakoro (Niger) », Bulletin de l'APAD [En ligne], 31-32 | 2010, mis en ligne le 26 juillet 2010, consulté le 07 septembre 2020. URL : http://journals.openedition.org/apad/4052 ; DOI : https://doi.org/10.4000/ apad.4052

Ce document a été généré automatiquement le 7 septembre 2020.

Bulletin de l'APAD 


\title{
Foncier, pouvoirs locaux et décentralisation dans le département de Dakoro (Niger)
}

\author{
Abdoulaye Mohamadou
}

1 Le processus de démocratisation amorcé au Niger depuis le début des années 90 s'est traduit, au plan politique par la mise en route d'un projet de décentralisation. Après un début laborieux lié en partie à l'instabilité politique du pays, la décentralisation est devenue effective avec le découpage territorial de 2001 et les élections locales de juillet 2004. Il s'agit d'une avancée démocratique majeure pour un pays longtemps dirigé par une dictature militaire.

2 Mais comme nous allons tenter de le montrer dans ce texte la décentralisation a eu pour conséquence le renforcement des chefferies locales (cantonales, villageoises et de groupements), la confirmation du primat de la sédentarité sur la mobilité et la marginalisation des éleveurs qui n'ont eu d'autres choix que de s'inscrire dans une logique de sédentarisation pour «obtenir» des chefferies et des "territoires" communaux. A long terme, cette évolution vers la territorialisation pourrait constituer une menace pour la survie du mode de vie pastoral dans les régions agricoles et agropastorales.

\section{Contexte}

3 La loi n60 du 30 avril 2002 portant création des communes et fixant le nom de leurs chefs lieux a découpé le territoire de la République du Niger en 265 communes (urbaines et rurales). L'Etat a décidé au nom d'une démarche progressive et prudente de ne rendre opérationnel que le niveau communal. Rappelons que les communes sont le dernier niveau de découpage qui comprend aussi dans l'ordre ascendant des départements et des régions. Ces collectivités territoriales administratives sont venues se superposer aux territoires coutumiers qui comprennent des sultanats, des provinces, des cantons et des zones pastorales. 
4 L'Etat a longtemps hésité sur le schéma de découpage à adopter craignant surtout que la décentralisation ne soit une source de velléité d'autonomie ou de sécession de certaines régions (Hahonou 2006). Finalement, c'est un découpage, basé sur les cantons et certains groupements, qui sera retenu. Les cantons sont de petites chefferies sédentaires créées par l'administration coloniale et qui regroupent quelques villages et tribus. Pour l'administration des nomades, ce sont des groupements qui ont été créés. Les cantons ont des territoires avec des frontières plus ou moins connues alors que les groupements n'en ont pas. Certaines chefferies cantonales ou de groupements ont une légitimité précoloniale mais la plupart sont des entités administratives mises en place pour les besoins du contrôle des populations (Olivier de Sardan 1998). Mais la question du découpage n'était pas la seule inquiétude, il y avait aussi la place que devrait avoir les chefs traditionnels dans la future configuration des pouvoirs locaux.

5 Le Niger est l'un des rares pays de l'Afrique de l'Ouest a avoir maintenu la chefferie traditionnelle comme un pilier de l'Etat poursuivant ainsi une pratique de l'Etat colonial. Au Mali et au Bénin par exemple où les chefferies ont été affaiblies par les régimes socialistes de Modibo Keita et de Mathieu Kérékou, la décentralisation est présentée comme une manière de «réconcilier le pays légal avec le pays réel » dans le cas du Mali et un moyen de revalorisation de la chefferie traditionnelle dans le cas du Bénin (Blundo 1998 ; Fay 2000).

6 La chefferie traditionnelle en particulier cantonale était au Niger en l'absence d'une expérience municipale, l'acteur politique dominant des arènes locales nigériennes. Mais cette situation a évolué au cours des vingt dernières années. La chefferie ellemême ne parle pas d'une seule voix. Elle comporte plusieurs lignages avec certains au pouvoir et d'autres dans l'opposition (Olivier de Sardan 1998). Depuis le début des années 80 avec la généralisation de l'approche projet, de nombreux comités villageois ont été mis en œuvre par les projets et ONG. Les dirigeants de ces projets sont devenus au fil des ans des notabilités locales qui tirent leur légitimité des liens avec les projets et $\mathrm{ONG}$ et des ressources matérielles et financières qu'ils contrôlent localement. Il peut s'agir d'anciens leaders des coopératives et de jeunes (samarias), organisations affiliées aux anciens régimes (du PPN-RDA et des militaires) ou de nouveaux acteurs souvent opposés aux chefferies.

7 La libéralisation politique amorcée depuis le début des années 90 a enfanté les partis ${ }^{1}$ politiques qui se sont implantés aussi dans les villages avec des leaders plus au moins importants en fonction des ressources (économiques, capital social, capital symbolique) qu'ils peuvent mobiliser. Ces leaders d'origines diverses peuvent être basés au niveau local, régional et national. Dans les deux derniers cas, ils agissent en tant que ressortissants, courtiers en développement et/ ou entrepreneurs politiques (Hahonou 2006).

8 Les pouvoirs locaux sont donc le produit de plusieurs processus interdépendants, comme la recomposition des espaces politiques précoloniaux suite à la pénétration française, et la superposition de nouvelles formes politiques mises en place pour assoir un pouvoir centralisé symbolisé par l'Etat colonial et l'Etat postcolonial (Bourmaud 1997, cité par Olivier de Sardan 2007).

9 La décentralisation démarre donc dans des paysages socio-historiques variés (Fay 2000) et des arènes complexes (Bierschenk \& Olivier de Sardan 1998). C'est pour cette raison que Blundo (1998:17) nous rappelle que «L'absence de terrains institutionnels 'vierges' est donc une donne essentielle pour comprendre la manière dont la décentralisation - 
qui vise à implanter de nouveaux modes de gestion, de nouvelles formes de légitimité et d'autorité et de nouvelles règles juridiques -, fonctionnera concrètement ».

Le processus de décentralisation a suscité un intérêt certain pour le local tant de la part de ses promoteurs (Etat et bailleurs de fonds) que des acteurs locaux et des chercheurs en sciences sociales. Au Niger l'Etat avait mis en place une administration de mission, le Haut Commissariat à la Reforme Administrative et à la Décentralisation (HCRAD) chargé de l'opérationnalisation du projet de décentralisation. Cette institution avait pour mission d'animer le processus en relation avec les différents acteurs concernés. Le choix d'un schéma de découpage basé sur les cantons et certains groupements a mis au devant de la scène les chefs traditionnels. Ces derniers étaient les principaux interlocuteurs du HCRAD au niveau local. Les administrateurs locaux et les services techniques ont été peu impliqués dans le processus.

11 Les bailleurs de fonds quant à eux s'étaient investis dans la décentralisation avant 2004 à travers les projets de développement local dont l'un des axes principaux était la bonne gouvernance déclinée par la mise en place d'institutions locales de concertation et de gestion des activités de développement.

12 Au niveau local, le projet de décentralisation a suscité beaucoup d'appréhensions (Mohamadou 2003) avant que sa réalisation ne mobilise les différents acteurs du pouvoir local. Les chefs traditionnels voyaient d'un mauvais œil l'arrivée des conseils municipaux avec lesquels ils auraient à partager leurs anciennes prérogatives et responsabilités : collecte des impôts, justice civile, développement local, gestion des marchés, gestion du foncier, etc.

La question foncière est certainement la problématique la plus délicate car son traitement ait toujours été un sujet éminemment politique au Niger. C'est une chasse gardée des chefferies traditionnelles sous le contrôle de l'Etat. Il était par exemple conseillé aux projets de ne pas intervenir là où il $\mathrm{y}$ a des problèmes fonciers. Les chefs traditionnels ont réussi à renforcer leur pouvoir sur le foncier à travers les textes de loi notamment l'ordonnance $\mathrm{n}^{\circ}$ 93-28 du 30 mars 1993 portant statut de la chefferie traditionnelle arrachée le dernier jour du régime de transition de l'après conférence nationale. Les textes du Code rural reconnaissent aussi le pouvoir des chefs sur le foncier et ils sont membres des commissions foncières.

14 La décentralisation apparaissait dès lors pour les chefs comme un risque de remise en cause de leurs privilèges en matière foncière mais le non transfert aux nouvelles communes de compétences dans le domaine foncier profite aux chefferies traditionnelles dont les prérogatives et attributions n'ont pas été abrogées. Les conseils municipaux vont se contenter de la compétence générale qui ne clarifie ni les pouvoirs, ni les responsabilités des uns et des autres. Fay $(2000: 123)$ résume bien le contexte dans lequel intervient la décentralisation marqué par une forte prégnance et imbrication du foncier et du politique.

«[...] du point de vue du paysan, tout s'inscrit dans des paysages socio-historiques variés, où coexistent des formes concrètes très différentes de solidarité d'une part (notamment autour des 'propriétés' possédées et/ou gérées à différents niveaux au nom d'appartenances ethniques, lignagères, villageoises ou historico-politiques), de pouvoir d'autre part (de la chefferie lignagère à la chefferie de village, à des rémanences de chefferies d'origine précoloniale ou coloniale, et aux pouvoirs administratifs et politiques de différentes natures) ». 
15 Ici se trouve posée la question de la réappropriation de la décentralisation par les acteurs locaux. La décentralisation qu'elle soit imposée ou produite de la nécessité historique a suscité des inquiétudes, des attentes, des stratégies d'anticipation, des négociations, etc. Le découpage des cantons, le choix des groupements nomades éligibles, le rattachement des villages et des tribus, le choix des chefs lieux des communes, le choix des noms des communes, etc. ont été l'objet de négociations, oppositions et compromis entre acteurs locaux et acteurs nationaux.

Dans cette première phase $d u$ processus, ce sont les chefs traditionnels, les ressortissants et les leaders associatifs qui ont joué un rôle important. La longue durée de gestation du projet (1994-2004) a permis aux différents acteurs d'élaborer des stratégies d'anticipation pour leur positionnement.

17 Nous menons des recherches ${ }^{2}$ à Dakoro depuis 2002, ce qui constitue une assez longue période d'observation des processus sociaux autour de la décentralisation. Notre approche s'inscrit dans une perspective historique et interactionniste pour comprendre les transformations et les permanences de l'arène politique locale. L'analyse va s'orienter sur deux axes. Dans un premier temps nous allons faire une genèse de la mise en ordre foncière dans le département en montrant que l'accès à la chefferie cantonale ou de groupement a toujours été pour les différents groupes le moyen privilégié de contrôle des populations dans une zone dont l'occupation massive de l'espace date de la période coloniale. Dans un second temps, nous analyserons le processus de construction des communes à travers les stratégies des différents acteurs dans le canton de Birnin Lallé et sa zone restante.

\section{La territorialisation/ appropriation de l'espace : des frontières aux usages}

18 La collectivité de Dakoro a été créée dans ses frontières actuelles en 1947 comme subdivision administrative rattachée au cercle de Maradi. Elle est née d'un redécoupage des subdivisions et cercles voisins et comprenaient quatre cantons (Kornaka, Birnin Lallé, Bader Goula et Soli Tagriss) et 3 tribus nomades (Serkin Rafi, Kel Gress et Kel Ferwan).

19 Nous allons faire l'historique de l'appropriation du «foncier-territorial » selon le mot de Fay (2000) depuis la création de la subdivision. Dans la nouvelle collectivité où la chefferie est peu institutionnalisée, le contrôle des populations et de l'espace vont de paire. Les nouveaux cantons créés n'avaient pas d'assise territoriale et n'ont pas de limites précises. Il fallait donc chercher le rattachement des entités villageoises qui disposaient de propriétés foncières. Les villages eux-mêmes, venus d'horizons divers s'étaient récemment installés et étaient très mobiles. Dans une étude sur la région de Gao, Grémont (2007) souligne que dans les zones peu peuplées, les groupes qui se disputent le pouvoir politique cherchaient d'abord à contrôler les populations. Le contrôle des hommes déterminait le contrôle de l'espace. C'est une situation très proche de celle de Dakoro.

20 Apres les rattachements des villages et tribus qui ont donné aux cantons et aux groupements leurs configurations territoriales actuelles, l'enjeu était devenu la mise en valeur des terres. La croissance démographique, les sécheresses récurrentes et les politiques foncières ont été les déterminants des pratiques et stratégies foncières. 


\section{Le découpage de $1947:$ " la zone restante », un espace de non droit} cantonales et chefferies nomades, entre éleveurs et agriculteurs. Alors que théoriquement, c'est un domaine public de l'Etat, diverses pratiques d'appropriation s'y sont développées. Les agriculteurs ont longtemps profité du flou juridique et du soutien tacite de l'Etat avant que les éleveurs ne se mobilisent à partir des années 90 pour une prise en compte $\mathrm{du}$ foncier pastoral dans la législation foncière nationale.

\section{De la limite nord aux terroirs d'attache : vers une reconnaissance du foncier pastoral}

En 1961, un an après les indépendances, le gouvernement du Niger a modifié le tracé de la limite des cultures en la repoussant plus au nord au profit des agriculteurs. C'est la loi № 61-05 du 26 mai 1961 (Journal officiel spécial 3 du 15/07/61) qui divise le pays en deux zones distinctes : une zone agricole au sud et une zone pastorale au nord. Au-delà de cette limite légale, l'agriculture est interdite et les dégâts commis sur des champs ne sont pas susceptibles de dédommagement. Seule l'agriculture oasienne et une agriculture de subsistance sont autorisées pour les nomades. Dans le département de

Bulletin de l'APAD, 31-32 | 2010 
Dakoro, cette limite se situe désormais au nord de la vallée de la Tarka à une vingtaine de kilomètres au nord du chef lieu du département. Mais la forte croissance démographique chez les sédentaires et les sécheresses des années 70 et 80 ont poussé à une colonisation des espaces pastoraux par les agriculteurs et des nomades qui ont perdu leur capital bétail. Le front agricole a franchi la Tarka et a pénétré dans la zone pastorale. Les couloirs de passage sont occupés et de nombreux conflits opposent agriculteurs et éleveurs autour des itinéraires de transhumance.

L'Etat a encouragé cette avancée du front agricole dans la mesure où le président de l'époque, Seyni Kountché a proclamé que «la terre appartient à celui qui l'exploite ». Or, l'élevage n'étant pas considéré comme une mise en valeur, le front agricole a progressé au nord au détriment de l'espace pastoral. Même l'adoption en 1993 d'une loi d'orientation sur les textes du code rural n'a pas corrigé cette lacune et les éleveurs se sont vus dépossédés de leur espace.

Dans la foulée de la libéralisation politique des années 90 , les éleveurs ont réagi avec la création de plusieurs associations pastorales dont l'un des objectifs majeurs était la défense des intérêts des éleveurs. Les affrontements meurtriers (une centaine de morts) de 1990 entre agriculteurs et éleveurs peuls dans le département de Guidan Roumji (frontalier de celui de Dakoro et par lequel passent plusieurs axes de transhumance en direction de la vallée de la Tarka) ont ému l'opinion nationale et ont servi à une prise de conscience de l'ampleur de la compétition autour du foncier.

La lutte menée par la société civile pastorale a abouti à l'adoption en 1997 d'une loi sur le Statut des Terroirs d'attache des Pasteurs. Cette loi stipule que les éleveurs ont des droits d'usage prioritaires sur leurs terroirs d'attache. Le terroir d'attache est défini comme une unité territoriale déterminée et reconnue par les coutumes et/ou les textes en vigueur, à l'intérieur de laquelle vivent, habituellement pendant la majeure partie de l'année, des pasteurs; unité territoriale à laquelle ils restent attachés lorsqu'ils se déplacent que ce soit à l'occasion de la transhumance, du nomadisme ou des migrations. Elle s'inspire fortement des droits coutumiers mais elle ne donne pas un droit de gestion et de négociation avec les tiers. Sa remise en cause par les associations des éleveurs est à l'origine du processus d'élaboration en cours d'une loi sur l'élevage et le pastoralisme. Mais ce texte constitue une avancée significative puisque la zone pastorale est désormais considérée comme un ensemble de terroirs d'attache des pasteurs et non plus comme des terres vacantes et sans maîtres. Les procédures administratives pour faire reconnaitre leurs droits sur les terroirs d'attache étaient lourdes, les éleveurs ont développé d'autres stratégies de marquage de l'espace.

\section{Les stratégies de marquage de l'espace des éleveurs}

31 L'objectif commun à tous les éleveurs (Peuls et Touaregs), c'est d'arrêter le front agricole. Plusieurs stratégies ont été observées dans la zone. Elles sont portées par les chefferies et le mouvement associatif pastoral. L'une des toutes premières stratégies a été de créer des ceintures agricoles autour des terroirs pastoraux. Les éleveurs euxmêmes se mettent à l'agriculture pour empêcher aux agriculteurs de défricher de nouveaux espaces. Ainsi un espace vital est préservé pour l'élevage. Certains villages d'agriculteurs sont rattachés aux chefferies nomades, ce qui permet à ces dernières de contrôler leur accès à la terre. 
32 Avec l'intervention des projets de développement de l'élevage dans la zone, les associations pastorales de concert avec les chefferies ont opté pour la création d'infrastructures socio-économiques (puits, écoles, banques céréalières, banques d'aliments du bétail, cases de santé, etc.). Il s'agit de créer des sites d'ancrage ou sites de fixation sur le modèle des villages d'agriculteurs pour marquer l'espace. Avec ces sites, une partie des familles ne vont pas en transhumance avec les animaux. Il y a donc une permanence d'occupation pour éviter l'arrivée de nouvelles populations. Par le passé la mobilité des éleveurs a favorisé l'installation de populations hausa qui ont fini par s'approprier l'espace en le cultivant. Ces deux stratégies combinées (ceintures de champs et viabilisation des sites d'ancrage) ont permis de stabiliser le front agricole autour de la vallée de la Tarka.

33 Avec le PASEL (Programme d'appui au secteur de l'élevage) financé par la coopération suisse, une action d'identification et de balisage des couloirs de passage et des aires de pâturage a été initiée. Elle a été conduite en concertation avec l'administration, les chefferies cantonales et nomades des départements de Dakoro et Guidan Roumji. Elle a permis de faire baisser les conflits entre agriculteurs et éleveurs. La commission foncière départementale (COFODEP), cheville institutionnelle du code rural nigérien a été maître d'œuvre du processus. La COFODEP dont la mission est de vulgariser les textes du code rural et sécuriser les opérateurs ruraux est composée de l'ensemble des acteurs du pouvoir local (Préfecture, services techniques, chefferies traditionnelles, société civile, éleveurs, agriculteurs, femmes et jeunes). Elle a joué un rôle important dans la prévention des conflits et la promotion d'une culture de concertation entre les différents acteurs. Le projet PASEL a aussi apporté un soutien aux associations pastorales qui se sont fédérées en 2001 en Collectif des Associations Pastorales du département de Dakoro (CAPONG). Il compte une dizaine d'associations animées principalement par des cadres et leaders peuls et touaregs. Ce collectif s'est distingué dans la défense des intérêts des éleveurs et s'était investi dans la mobilisation des fonds au profit des communautés pastorales. Il a réussi à s'imposer comme un acteur incontournable dans l'arène locale ${ }^{3}$.

Les investissements réalisés ont permis de doter les sites des principales tribus d'infrastructures sociales et hydrauliques. Le collectif s'est employé à défendre les intérêts des éleveurs dans le cadre du processus de décentralisation. Il a mené plusieurs campagnes de sensibilisation pour prévenir la marginalisation des éleveurs. Plusieurs de ses membres se sont impliqués et se sont fait élire conseillers municipaux.

Les positions institutionnelles jouent un rôle important dans le jeu politique local. Ainsi, nous verrons comment la quête de reconnaissance politique par les différents groupes sociaux a été une constante dans l'émergence des chefferies traditionnelles de Dakoro. La particularité de Dakoro par rapport à de nombreuses régions du pays, c'est l'absence avant la colonisation de chefferies coutumières. C'est à l'administration coloniale et à l'Etat postcolonial qu'il était revenu la responsabilité de créer des chefferies au sein de groupes récemment installés.

\section{Emergence des chefferies sur des « terres vacantes »}

Les populations actuelles de Dakoro appartiennent à divers groupes socio-linguistiques du pays et se sont installées au début du XXème siècle. La colonisation a joué un rôle majeur dans l'occupation progressive de l'espace de la nouvelle subdivision. 


\begin{abstract}
"A l'époque pré-coloniale, ce fut un 'no man's land', avec peu de ressources en eau, situé entre les territoires de plusieurs confédérations : en particulier Kel Gress, Kel Ferwan et Iwellemenden.[...] Le creusement de puits jusqu'à une profondeur de 30 à 40 mètres, à partir de cette époque, a permis durant toute l'année l'occupation de ces terres qui auparavant n'avaient été habitées qu'en saison des pluies. Des familles appartenant à différentes confédérations touarègues et à d'autres groupes ethniques - Arabes, Peuls (Fulani), Hausa - ont progressivement migré vers cette région durant les années trente et quarante, encouragées par une administration qui souhaitait démanteler les groupements politiquement les plus importants ». (Oxby 1996)
\end{abstract}

Avant la création de la subdivision, la région était une zone refuge pour les populations fuyant les exactions coloniales et l'arbitraire des chefs locaux. Mais il y a eu surtout une migration économique massive des populations hausa, touareg et peul à la recherche des terres des cultures et des pâturages. La majorité des villages au sud de la Tarka ont été créés entre 1930 et 1950 (Rey 1989).

Les populations étaient venues de toutes les zones limitrophes:

- les Hausa, groupe majoritaire est composé de deux groupes : les Gobirawa venus du sud et du sud ouest (du Gobir et de Madaoua) et les Aderawa venus de l'ouest (de Bouza principalement) ;

- les Touaregs étaient venus de l'ouest pour les Kel Gress (de Madoua), de l'est pour les Tagamaoua et les Kel Ferwan (de l'Aîr), du nord et nord ouest pour les Iwellemenden (de l'Azawagh);

- Les Peuls viennent du sud pour les deux groupes « Farfaru » et Wodaabé (de Sokoto).

Ces différentes populations se sont séparées des chefferies auxquelles elles appartenaient pour aller fonder de nouveaux villages et de nouvelles entités politiques. Le contexte de la colonisation a accéléré les processus de fractionnement des grands ensembles politiques.

\title{
Les chefferies coloniales : s'assurer le contrôle des populations
}

La création de la subdivision de Dakoro et de son chef lieu fut l'oeuvre de l'administrateur colonial Maurice Vilmin. Quand on parle localement de Dakoro, on ajoute souvent «Garin Maïbougé » c'est-à-dire village de Maïboué pour signifier qu'il en est le fondateur. Il était réputé pour ses méthodes arbitraires et la figure de commandant autoritaire qu'il a incarnée est restée vivace dans la mémoire collective.

Pour s'assurer le contrôle des populations de la nouvelle subdivision, l'administration coloniale met en place de nouvelles chefferies en détachant progressivement les populations des anciennes tutelles chefferiales précoloniales ou coloniales.

\section{La chefferie cantonale de Birnin Lallé}

La région nord de Maradi qui englobe une bonne partie du département actuel de Dakoro était pendant les premières années de l'occupation coloniale administrée depuis Kornaka, Maradi et Tanout. Le secteur de Dakoro actuel en particulier dans sa partie septentrionale qui nous intéresse était une zone excentrée et peu peuplée. Birnin Lallé était le seul gros village de la zone, avec un marché et une mare semi-permanente. Le village était devenu un lieu d'escale pour les convois de l'administration coloniale sur la route Maradi-Tanout. Mais le village de Birnin Lallé doit sa renommée aussi à son 
passé. En effet, Birnin Lallé est une ancienne capitale du Gobir historique (Hama 1967). Les vestiges de l'ancienne cité sont encore visibles à quelques mètres de l'actuel village. Elle a été abandonnée lors de la pénétration des Gobirawa vers le Sud. Dans les années 20, quelques familles venues de la région de Tibiri (fief actuel du Gobir) se sont installées dans le village ${ }^{4}$.

En 1931, Birnin Lallé est érigé en subdivision englobant les villages situés au nord de Birnin Lallé et de Dakoro mais l'administration coloniale n'installa pas de poste administratif. Quand en 1947, il fut décidé enfin de créer un poste administratif, on l'implanta à Dakoro qui devint le chef lieu de la subdivision.

L'administration coloniale réorganisa les chefferies et trois nouveaux cantons ont été créés :

- le canton de Birnin Lallé avec comme chef lieu Birnin Lallé créé le 5 novembre 1947 ;

- le canton de Bader Goula avec comme chef lieu Goula ;

- le canton de Soli Tagriss avec comme chef lieu Soli.

Les Kel Gress d'Azagor et les Kel Ferwan de Gadabeji deviennent des tribus indépendantes. Ces nouvelles chefferies viennent s'ajouter à celle déjà existante du canton de Kornaka qui administrait le sud mais aussi les nomades de la zone dont la tribu peule Serkin Rafi. Le canton de Kornaka a été détaché de Maradi pour être administré par Dakoro.

Au cours de la même année 1947, les premiers chefs de cantons sont nommés après des élections. A Birnin Lallé, les élections du 3 novembre 1947 donnent la victoire à Boubakar Garandam. Il a été élu avec 32 voix sur 54 chefs de villages consultés. Son concurrent le plus sérieux était Haido Tambari Jackou, fils du chef de canton de Kornaka qui a recueilli 16 voix. Boubakar Garandam a été désigné chef de canton de Birnin Lallé le 12 décembre 1947 par le Gouverneur Toby lors de la visite de ce dernier dans la subdivision (Vilmin 1947).

La décision est confirmée le 2 janvier 1948 (Journal officiel de la colonie du Niger, $1^{\text {er }}$ février 1948, 203 :23). Par la même décision, Tambari Djako, chef du Kornaka assurera le commandement supérieur de l'ensemble des cantons de Kornaka et Birnin Lalle. Malgré la création du canton de Birnin Lalle, celui-ci reste sous la domination du canton de Kornaka qu'on appellera d'ailleurs province de Kornaka et de Birnin Lalle. Les autres chefs de canton de la subdivision ont également été nommés Tankari Dan Maika et Aghali Abambacho deviennent respectivement chefs des cantons de Soli Tagriss et de Bader Goula. Mouloul est nommé chef de la tribu des Kel Gress d'Azagor.

Le premier chef de canton de Birnin Lalle, Boubakar Garadam est originaire de l'Ader dans la région de Tahoua. C'est un Hausa Aderawa. Il était un dilali (courtier), commerçant et féticheur très influent, qui s'était lié d'amitié avec les agents de l'administration coloniale qu'il hébergeait lors de leurs escales à Birnin Lallé. Ses liens privilégiés avec l'administration coloniale et son capital économique ont probablement joué un rôle important dans son élection-nomination. Boubakar Garandam règnera de 1947 à 1972. A sa mort, il sera remplacé par Maydabo, du groupe Gobirawa de Birnin Lallé, issu de l'une des premières familles à s'installer dans le village. C'est un ancien goumier (les goumiers constituaient le corps d'auxiliaires indigènes méharistes mis en place par l'administration coloniale, chargés entre autres de la collecte des impôts). Il doit sa nomination, selon les descendants de Garandam, à son amitié avec un ancien administrateur civil du cercle de Tanout sous les ordres duquel il a servi. A la mort de 
Garandam, cet administrateur était préfet de Maradi sous le régime du Rassemblent Démocratique Africain (RDA). Après sa nomination, Maydabo quitta Birnin Lallé et s'installa à Dakoro.

49 La chefferie passe donc des mains du groupe des Aderawa à celui des Gobirawa. Maydabo meurt en 1981 et son fils Issoufou Maydabo, actuel chef de canton, est nommé par arrêté du président Seyni Kountché en sa qualité de ministre de l'intérieur. Il appartient au corps de la garde républicaine (nouvelle appellation du corps des méharistes) comme son père. Depuis que la chefferie est passée aux mains des Gobirawa, elle a progressivement réintégré le giron du Gobir en se rapprochant politiquement et symboliquement de la chefferie de province de Tibiri, qui assure la tutelle des chefferies des cantons du Gobir, et en s'appropriant les attributs et les rites des chefferies du Gobir, notamment la nomination d'une "Inna » ou « mère » (associée aux cultes de possession) et la présence d'un may raya, intronisateur (« celui qui passe le turban au chef ») désigné par le chef de province du Gobir.

Olivier de Sardan (1998: 67) explique comment les chefferies administratives coloniales essayent d'asseoir leur légitimité en se parant des attributs des chefferies précoloniales: "Tout un apparat et toute une idéologie produisent et reproduisent cette légitimité soi-disant 'traditionnelle', avec diverses 'inventions' de la tradition (ou néo-traditions) et le recyclage de certains éléments des rituels politiques précoloniaux, insérés dans un contexte tout différent ".

51 En se donnant une légitimité de chefferie du Gobir, les deux chefs de canton successifs visent surtout à disqualifier les autres groupes sociaux qui revendiquent la chefferie (les Aderawa et les Touaregs). Les candidatures aux premières élections de 1947 montrent que c'était un fauteuil ouvert.

52 La chefferie de Birnin Lallé est donc une chefferie administrative avec une faible profondeur historique. L'actuel chef est seulement le $3^{\text {ème }}$. Mais le symbole historique (ancienne cité du Gobir), le rattachement du village au canton de Kornaka au début de la colonisation et les origines de son premier chef de canton en font une chefferie disputée par plusieurs chefferies et groupes socio-linguistiques: la chefferie de Sabon Birni au Nigéria, qui était avant sa conquête par Ousman Dan Fodio la capitale du Gobir ; la chefferie du canton de Tibiri, actuelle capitale politique du Gobir nigérien, la chefferie de Kornaka, ancien chef lieu de la province de Kornaka et de Birnin Lallé et les descendants du premier chef de canton. Après la mort du second chef de canton en 1972, toutes ces chefferies avaient manifesté leur intention de présenter des candidats avant que le président Seyni Kountché ne décide de nommer par arrêté le nouveau chef de canton.

53 L'opposition à la chefferie est constituée par la famille de Garandam. Une grande partie de ses descendants et sympathisants est regroupée dans le quartier Kourmi à Dakoro. Ils sont propriétaires fonciers mais n'ont pas d'ancrage dans les zones rurales. Le groupe des Aderawa auquel ils appartiennent est surtout présent dans la ville de Dakoro, où il contrôle une grande partie de l'activité économique et investit de plus en plus dans la politique locale.

54 Mais les appréhensions du chef de canton s'orientent surtout vers l'émergence de nouveaux pouvoirs et de nouveaux acteurs avec la décentralisation. Dans ces conditions, son souci est de contrôler le processus de découpage pour amoindrir les effets de la territorialisation sur son pouvoir. 


\section{Le Serkin Rafi de Korahane} Wodaabé (grands transhumants) et les Peuls Katsinawa (animistes et hausaphones). Les nouveaux venus avaient surtout la particularité d'être d'obédience islamique et auréolés du prestige politique de Sokoto. Il faut dire aussi que les Peuls de Sokoto et les Touaregs Kel Gress de Kornaka entretenaient de vieilles relations politiques. Les Tambari de Kornaka étaient intronisés pendant les premières années du XIXème siècle à Sokoto (Jackou 1970).

58 Au début des années 20, avec la dislocation par l'administration coloniale de la chefferie peule de Mahaman Assao qui assurait le commandement des Peuls de Maradi, ces derniers ont été subdivisés en trois chefferies dans les trois cantons sédentaires de Maradi, du Gobir et du Kornaka.

«En 1924, le chef de canton de Kornaka reçut le commandement des 11 fractions peules nomadisant dans son canton. Il laisse peu à peu le commandement de ce groupement à un de ses Ardo Serkin Rafi Amadou, originaire du Nigeria et ayant séjourné vers Madaoua en 1925 » (Jackou 1970). 1946 plus au nord à Korahane, leur fief actuel, à la suite de malentendus entre Amadou Serkin Rafi et Tambari Jackou. devient chef de groupement. Il mourut en 1984 et son fils Mahé (actuel chef) lui succéda. Celui-ci a deux résidences, une à Korahane, chef lieu du groupement, et une seconde à Dakoro. Le groupement était jusqu'en 1980 à la tête de plus d'une centaine de tribus peules et touaregs. La création dans les années 80 de trois nouveaux groupements, le groupement Wodaabe de Bermo, le groupement Kel Gress d'Azagor et le groupement Kel Ferwan de Gadébéji, l'a amputé de la plus grande partie des tribus qui lui étaient rattachées. Il ne dirige aujourd'hui que 18 tribus. 
La stabilisation du front agricole et la préservation d'un espace vital pour l'élevage et le groupement sont un nouvel enjeu pour le Serkin Rafi. La communalisation apparaît comme une opportunité pour soustraire le secteur de la tutelle politique du canton de Birnin Lallé.

\section{Les chefferies postcoloniales}

63 A l'indépendance en 1960, le canton de Birnin Lallé et « la zone restante » comptaient trois chefferies principales : le canton de Birnin Lallé, le groupement Serkin Rafi et la tribu indépendante Kel Gress Mouloul.

\section{Le groupement Kel Gress d'Azagor}

64 région de Madaoua au début du siècle. Ils appartiennent à la confédération touareg des Kel Gress, mais se sont métissés au cours des années avec les populations locales et l'autre groupe touareg du departement, les Kel Ferwan, venus de la région d'Agadez.

65 A l'arrivée des Français, ils nomadisaient déjà dans la région. Un village symbolise leur présence, Maylafia, situé à une vingtaine de kilomètres au nord-est de Dakoro. C'est ce village qui a accueilli en 1947 la première école nomade de la région, qui a formé les premiers cadres ${ }^{5}$ touaregs et peuls de la région dont trois dirigent des partis politiques à Niamey. Dans le cadre de la politique de recensement et de contrôle de la population par l'administration coloniale, les Kel Gress devinrent une tribu indépendante. Cette tribu que dirigeait le père de l'actuel chef de groupement au moment de la création de la subdivision de Dakoro a entretenu des relations sans histoires avec l'administration coloniale (Vilmin 1947).

66 même temps que celles des Kel Ferwan et des Peuls Wodaabe. Ces promotions ont été possibles grâce à la mobilisation des réseaux sociaux et politiques au sein du régime militaire notamment les préfets militaires successifs de Maradi et des cadres ressortissants évoluant dans les hautes sphères de l'Etat, car le népotisme ethnique est une pratique constante des régimes nigériens successifs.

La plus grande partie des membres du groupement Kel Gress sont sédentarisés dans des villages appelés localement Zongo. Certains de ces Zongo sont situés sur le territoire d'autres cantons et groupements du département. Mais la plupart des chefs de tribu sont installés autour du chef lieu du groupement, le campement d'Azagor. du canton de Birnin Lallé. Il est selon lui dans la zone restante. Il défend la spécificité pastorale de son fief, dans la vallée de la Tarka. Tout comme le Serkin Rafi, l'enjeu principal pour le groupement d'Azagor est le contrôle de l'accès au foncier et aux ressources naturelles. Le contrôle d'une commune rurale, au-delà de l'enjeu politique, est perçu surtout comme un moyen d'obtenir un droit de gestion sur les ressources pastorales.

Lorsque la tribu voulait devenir groupement, le premier chef avait autorisé l'installation des agriculteurs qu'il faisait recenser pour augmenter le nombre de ses administrés. Cette stratégie se retourne aujourd'hui contre le groupement, les agriculteurs ayant colonisé une bonne partie de l'espace pastoral du groupement et ont 
fondé des villages dont certains sont administrativement rattachés au canton de Birnin Lallé.

Compte tenu du caractère récent de l'occupation de l'espace et de la création des chefferies, on observe qu'il y a un enchevêtrement des populations, une instabilité et une pluralité de tutelles administratives. Les chefs des villages passent d'une chefferie à une autre au gré de leurs intérêts. Certains sont affiliés à deux ou trois chefferies à la fois.

La faible profondeur historique des chefferies et la mobilité des populations aussi bien nomades que sédentaires engagées dans une compétition d'occupation d'un espace «vacant» explique ce phénomène. Le plus souvent, ce sont plusieurs familles avec leurs chefs qui se déplacent à la recherche de nouvelles terres.

La création des trois groupements a renforcé le poids politique des nomades et leurs assises territoriales dans le département de Dakoro. Même si les ressources pastorales demeurent ouvertes, dans la pratique les groupements exercent un contrôle sur des portions de la zone pastorale.

\section{Les chefferies de l'après communalisation}

La zone pastorale de Dakoro est occupée par plusieurs sous-groupes Peuls et Touaregs. La création des premiers groupements a permis aux tribus Kel Gress et Kel Ferwan de mettre sous tutelle les autres tribus touaregs et au clan peul du premier chef de prendre de l'ascendant sur les autres lignages des Wodaabé.

La politique de création de groupements remise à l'ordre du jour sous la $5^{\text {ème }}$ république (depuis 2000) a offert une opportunité à d'autres groupes de nomades du département de Dakoro de revendiquer des groupements. L'option de création de nouveaux groupements s'inscrit dans une politique visant à donner un cadre d'expression politique et une assise territoriale aux éleveurs peuls. Ces derniers, pour la plupart, transhumants n'avaient pas de chefferies de nouveau groupement, ce qui les excluait de la gestion politique locale. Par la suite, cette politique a été étendue aux autres groupes nomades (Touaregs, Toubous et Arabes) souvent pour des raisons clientélistes et électoralistes.

Dans le nord Dakoro, deux nouveaux groupements ont été créés :

- Le groupement touareg des Kel Temerkest. Les Kel Temerkest viennent de l'Azawagh au nord et appartenaient à la confédération des Iwellemenden. Ils étaient rattachés au 2 ème groupement d'Abalak avant de migrer dans le nord de Dakoro. Ils se sont rattachés au Serkin Rafi d'abord, et au groupement Kel Gress, ensuite ;

- Le groupement Peul Kassassawa. C'est un important lignage des Woddabé qui s'est autonomisé de la première chefferie.

Ces deux groupements sont localisés dans l'actuelle commune de Bermo avec des administrés disséminés dans l'ensemble de la zone pastorale dite restante. Leur création est la conséquence des conflits autour de l'occupation de l'espace entre différents groupes d'éleveurs et la volonté de certaines tribus ou lignages de participer au jeu politique local. Au cours des dernières années de nombreux conflits ont opposé Peuls et Touaregs mais aussi sous groupes peuls entre eux et sous groupes Touaregs entre eux autour du fonçage de points d'eau (Mohamadou 2004). 
77 L'émergence des deux chefferies a fragilisé les premières chefferies nomades qui ont perdu une partie des tribus qui leur étaient rattachées. Comme les premières, les nouvelles chefferies ont opté pour une politique de marquage de l'espace (villagisation, fonçage de puits, courtage, etc.) qui préfigurent une tendance à la territorialisation. On peut faire l'hypothèse qu'elles finiront par réclamer dans les années à venir des communes à elles.

\section{La construction des communes : entre critères officiels et logiques sociales}

La popularisation du projet de décentralisation a été faite dans le cadre de missions ministérielles et parlementaires. Elles ont surtout concerné les leaders d'opinion au niveau des chefs lieux des départements. A la suite de la promulgation du projet de loi sur la décentralisation, il a été demandé aux chefs de cantons et de groupements de faire des propositions de communalisation de leurs territoires. Les propositions sont envoyées au Haut Commissariat à la réforme administrative et à la décentralisation qui les examine, les amende et envoie des missions sur le terrain pour en discuter avec les acteurs locaux.

79 Il n'existait pas au niveau local un cadre de concertation autour du projet de décentralisation, ce que dénonçaient à Dakoro le sous-préfet et les responsables des services techniques qui considéraient que le découpage obéissait plus à des logiques politiques que techniques. Pour eux, les critères techniques et économiques ne sont pas encore pris en compte alors qu'ils déterminent la viabilité économique et financière des futures communes. La symbolique du pouvoir a pris le pas sur les enjeux économiques.

Le principe de la communalisation des cantons et de certains groupements a laissé la porte ouverte à toutes les interprétations, aux négociations et compromis. Pour les chefs des cantons et groupements, le découpage, c'est leur affaire puisque les territoires et les populations sont sous leur responsabilité. Il y a derrière cette perception, la volonté des chefs de mettre sous tutelle cantonale les futures communes rurales. La loi leur a du reste ouvert le chemin puisqu'ils sont membres de droit avec voix consultative de tous les conseils municipaux des communes créés sur leurs entités territoriales ou administratives. Les chefs lieux des cantons et de groupement doivent naturellement devenir des chefs lieux des communes. Dans l'ensemble du département de Dakoro, tous les sièges de chefferies de rang cantonal sont devenus chefs lieux de communes à l'exception du canton de Soli Tagriss où un puissant leader politique national a transféré le chef lieu de la commune dans son village. Les critères technicoadministratifs ${ }^{6}$ du HCRAD apparaissent comme des possibilités de légitimation parmi plusieurs autres mobilisées localement: légitimités historiques, capital social, légitimité politique, etc.

\section{Le découpage du canton de Birnin Lalle}

81 Le canton de Birnin Lalle compte 4 grands secteurs avec comme épicentre la ville de Dakoro. Un secteur ouest autour du village de Korahane, un secteur nord avec comme village principal, Intuila, un secteur nord-est autour du marché de Sakkabal, un secteur sud-est avec Birnin Lalle comme centre politique. Les secteurs sont organises autour de 
villages centres abritant soit une importante chefferie villageoise, soit un marché hebdomadaire, soit des infrastructures scolaires ou sanitaires.

La première proposition du chef de canton était de 12 communes. Tous les gros villages du canton ont demandé à être érigés en communes. C'est cette proposition qui a été transmise au HCRAD qui n'a retenu que trois communes avant que l'Assemblée Nationale n'en rajoute une quatrième. Voyons le cas des communes retenues et quelles sont les logiques qui ont prévalu.

\section{La commune rurale de Birnin Lallé : le poids du symbole}

Sa création a été surtout dictée par des considérations historiques et politiques car elle ne satisfait à aucun des critères officiels. Elle a pour elle la légitimité historique en tant qu'ancienne cité historique du Gobir. Pour le chef de canton, c'est pour la charge symbolique qu'il fallait la créer. Mais, de son passé glorieux, il ne reste plus que les vestiges, au point où le village ne compte comme infrastructures qu'une école. Le marché ne s'anime plus; le chef de canton a installé son palais depuis 1972 à Dakoro, ville qui n'est distante que d'une dizaine de kilomètres qui abrite le chef lieu de la commune urbaine.

D'autres gros villages de la commune ont contesté le choix de Birnin Lallé comme chef lieu de la commune rurale à cause de sa position géographique et de son faible équipement. Les ressortissants d'Intuila ont vivement protesté parce que leur secteur satisfaisait aux critères officiels. Finalement, leur secteur a été éclaté entre la commune rurale de Birnin Lalle qui voulait l'englober et la commune urbaine de Dakoro dont le siège est plus proche et qui a la préférence des communautés. Cinq villages sont actuellement en litige entre les deux communes mais, tout porte à croire qu'ils seront intégrés dans la commune urbaine de Dakoro. L'absorption par cette dernière commune du marché de Intuila et par la commune de Roumbou1 de celui de Sakkabal a privé la commune de Birnin Lallé de gisements fiscaux. La commune de Birnin Lalle apparait comme la commune du fief premier du canton sur lequel la chefferie exerce un contrôle en raison de la proximité sociale de ses habitants.

\section{La commune rurale de Korahane : un espace très disputé}

Le village de Korahane est situé à $23 \mathrm{~km}$ à l'ouest de Dakoro en bordure de la Tarka sur la route latéritique Dakoro-Keita. Il a été fondé par un chasseur hausa du nom de Nahantchi. Avant la création du village, des nomades peuls wodaabé et touaregs nomadisaient dans la région. Le fonçage du premier puits a été l'œuvre de Peuls Wodaabé, ce qui constitue une forme de marquage de l'espace chez les nomades.

Quatre chefferies sont installées dans le village :

- la chefferie du village de Korahane I, qui regroupe les descendants du fondateur du village;

- la chefferie du village de Korahane II, dont le chef Chipkao est arrivé avec sa population de Sourakane, un village situé à $4 \mathrm{~km}$ plus à l'Ouest, suite à une dispute avec des Peuls. Bien que d'un groupe social casté, sa forte personnalité lui a permis de supplanter le premier chef, - la chefferie du groupement peul Serkin Rafi qui s'est installé aux abords du village,

- la chefferie du village peul, ardo Dodo, nommé par le Serkin Rafi, dont les administrés viennent d'un fractionnement des populations des deux premiers villages. C'est un épisode dans la rivalité entre les chefferies sédentaires et la chefferie nomade. 

premier ministre de la transition civile de 1991-1993 est actuellement président d'un parti politique, le RSD-Gaskiya ${ }^{7}$ qui est né d'une scission de la CDS-RAHMA ${ }^{8}$. Il est président du Conseil économique social et culturel, une institution de la république qui conseille le gouvernement. Korahane est un fief du parti. Il recrute principalement dans la communauté peule alors que les Hausa gobirawa, l'autre groupe du village votent massivement pour le MNSD-Nassara9. Aux élections locales du 24 juillet, les deux partis ont obtenu chacun 5 conseillers (soit 10) sur les 11 postes en jeu.

91 La rivalité autour des ressources naturelles se double donc d'un duel politique animé par les ressortissants. Le MNSD est porté localement par un technicien en télécommunication, petit-fils du premier chef de village qui a du reste été élu maire de la commune.

\section{La commune rurale de Roumbou I : la revanche des Touaregs Ibroubak}

Elle faisait partie des 12 premières communes proposées au titre du canton mais elle ne figurait pas sur la liste des communes transmises à l'Assemblée Nationale. Ce secteur du 
canton situé en zone agropastorale autour du grand marché de Sakkabal est occupé par des touaregs Ibroubak agropasteurs et des agriculteurs hausa. Toutes les deux communautés sont administrées par le chef de canton de Birnin Lallé. Ici les Touaregs se sont sédentarisés très tôt et détiennent des droits fonciers sur les terres au même titre que les agriculteurs hausa.

Lorsque que les ressortissants touaregs de l'aristocratie villageoise ont appris que leur secteur n'avait pas été retenu comme commune, ils ont adressé une lettre de protestation au HCRAD. Ils accusent indirectement le chef de canton d'avoir enlevé le nom de leur secteur dans les propositions. Pour eux, c'est une manière d'affaiblir la chefferie villageoise.

Un ressortissant de la chefferie villageoise de Roumbou qui était à l'époque député a négocié pour ajouter la commune de Roumbou avant l'adoption de loi déterminant le nombre et les noms des chefs lieux des communes. De nombreuses communes ont été créées sur l'initiative des membres du gouvernement et des députés. Le nombre proposé par le HCRAD a été pratiquement multiplié par deux.

Le gros village de Sakkabal avec son marché n'a pas été choisi comme chef lieu de la commune. Le député a préféré donné le nom du village de sa famille à la commune. Il est distant de quelques mètres de Sakkabal.

La création de la commune de Roumbou I est considérée par ses ressortissants comme une reconnaissance politique des Touaregs Ibroubak. Ces derniers estiment avoir été spoliés par l'administration coloniale de leur pouvoir politique au profit des autres groupes Touaregs et Hausa du département. Au-delà de la légitimité historique retrouvée, les Touaregs Ibroubak de Roumbou ont mis fin à leur façon à la rivalité entre le chef de canton de Birnin Lallé et le chef de groupement Kel Gress d'Azagor. Ces deux notabilités convoitaient les villages de ce secteur et surtout le marché de Sakkabal. La création de la commune de Roumbou montre aussi le rôle des ressortissants dans les liens entre le national et le local.

\section{La commune urbaine de Dakoro}

97 Le chef lieu de l'arrondissement de Dakoro (devenu département en 2004) a été érigé en commune urbaine par la loi n096/98 mais il n'y a jamais eu d'élection pour élire le conseil municipal. Il a fallu attendre 2004 pour que la commune urbaine soit dotée d'un territoire et d'un conseil municipal élu. Bien que située sur son territoire et abritant son siège, la chefferie cantonale a une faible emprise sur la ville de Dakoro. Son statut de centre administratif abritant le pouvoir d'Etat, le cosmopolitisme de son peuplement et la présence de l'élite économique et intellectuelle du département aux origines diverses en font un espace presque autonome.

98 La ville est constituée de plusieurs quartiers-villages regroupant tous les groupes sociaux du département: les Hausa gobirawa, les Hausa aderawa, les Touaregs Kel Gress, les Touaregs Roumboukawa, les Peuls, etc. Une partie des chefs de quartiers ne dépend pas du chef du canton. Mais ce dernier a tenté de circonscrire la zone rurale de la commune urbaine. Il s'est heurté à l'opposition des communautés de la zone périurbaine qui ont préféré se rattacher à la commune urbaine plus proche et qui offre plus de services.

99 La fondation des communes du canton s'est faite selon des critères différents. Le chef de canton a réussi à faire du siège historique de la chefferie un chef lieu de commune 
contre les critères officiels. Sa tentative de créer une grande commune rurale regroupant deux autres secteurs (Intuila et Sakkabal) qui allaient assurer la viabilité économique de la commune a échoué. Il pourrait ainsi perdre le contrôle politique sur une partie de la population du canton. La création de la commune de Roumbou ressuscite un lignage aristocratique touareg longtemps mis aux marges de la politique locale. Quant à la commune de Korahane, son émergence pourrait renforcer la chefferie du groupement peul.

\section{La commune du groupement Kel Gress d'Azagor}

Le village d'Azagor est situé à une vingtaine de kilomètres au nord de Dakoro. C'est le chef lieu du groupement Kel Gress. Le village dispose d'infrastructures dont la réalisation a été financée par le Projet de développement de la zone pastorale (PROZOPAS) initié dans le cadre des accords de paix de 1995 entre l'Etat du Niger et la rébellion touareg. Il s'agit d'une école et sa cantine, d'une case de santé, d'une banque céréalière et d'un parc de vaccination. Il existe deux puits cimentés l'un construit par l'Etat et l'autre par le projet « Hydraulique villageoise ».

Le choix du chef lieu du groupement comme chef lieu de la commune n'a pas posé problème. Le village est le seul à répondre aux critères officiels sans compter le leadership du chef de groupement. Mais le chef de groupement n'a pas réussi son projet de fédérer les Touaregs de la Tarka de Dakoro dans une seule commune. Une partie des tribus et Zongo du groupement a choisi de se rattacher à la commune urbaine de Dakoro. La création de la commune de Roumbou dont le chef lieu est à $13 \mathrm{~km}$ d'Azagor a été mal ressentie. Elle prive Azagor de plusieurs villages qui étaient prêts à se rattacher et du marché de Sakkabal. Des vieilles mésententes entre les deux groupes touaregs autour de l'implantation des infrastructures socio économiques seraient à l'origine de l'échec du projet de création d'une commune unique.

\section{Conclusion}

Le choix de l'Etat du Niger d'un découpage basé sur les cantons et certains groupements a cristallisé les enjeux politiques autour du local. La superposition de territoires administratifs et de territoires coutumiers a réveillé de vieilles rivalités entre les notabilités locales et entre les agriculteurs et les éleveurs. Ces derniers tentent de profiter de la décentralisation pour baliser les frontières de leurs territoires dans une zone agropastorale très disputée aux agriculteurs. La décentralisation au-delà du découpage territorial pose aussi le problème de la construction de l'Etat. Ce dernier était absent des zones rurales et la fourniture des services et biens publics a fortement régressé ces dernières années du fait des difficultés économiques. L'Etat avait délégué aux chefferies la gestion des affaires politiques et économiques locales mais celles-ci gouvernent selon un mode patrimonial et "extractif» et ne sont pas porteuses de projet de société. Elles sont surtout préoccupées par la préservation de leurs privilèges. Avec l'émergence des communes, le maillage administratif s'améliore et il faut s'attendre à de fortes rivalités entre les élus locaux et les chefs traditionnels sous l'arbitrage de l'Etat central et des partis politiques. Au Benin et au Mali, la décentralisation a réhabilité et renforcé les chefferies. Au Niger, les chefferies ont des 
appréhensions sur leur avenir, ce qui explique leur opposition à la décentralisation et leur tentative d'influencer le jeu politique au niveau national.

Bierschenk T., J.-P. Olivier de Sardan 1998. Les arènes locales face à la décentralisation et à la démocratisation : Analyses comparatives en milieu rural béninois. InLes pouvoirs au village : le Bénin rural entre démocratisation et décentralisation (eds) T. Bierschenk \& J.-P. Olivier de Sardan. Paris : Karthala.

\section{BIBLIOGRAPHIE}

Blundo, G. 2000. Décentralisation et pouvoirs locaux : Registres traditionnels du pouvoir et nouvelles formes locales de légitimité (Atelier : II), Le bulletin de l'APAD 16, 147-150 :

« Décentralisation, pouvoirs locaux et réseaux sociaux » (eds) G. Blundo \& R. Mongbo.

Fay, C. 2000. La décentralisation dans un cercle (Tenenkou, Mali). Autrepart 14, 121-142.

Grémont, C. 2007. Du contrôle des hommes et du contrôle de l'espace : Nouvelles formes de pouvoir et décentralisation dans la région de Gao (Nord Mali). Communication présentée au colloque international sur les pouvoirs locaux, LASDEL, Niamey, octobre 2006.

Hama, B. 1967. Histoire du Gober et de Sokoto. Paris : Présence africaine.

Jackou, S. 1970. Intégration économique et sédentarisation des populations nomades : cas du Niger. Thèse, Université Paris I. Institut d'études pour le développement économique et social.

Le Roy, E. 1999. A la recherche du paradigme perdu : Le foncier pastoral dans les sociétés sahéliennes. In Horizons nomades en Afrique Sahélienne : Sociétés, développement et démocratie (ed.) A. Bourgeot. Paris : Karthala.

Mohamadou, A. 2003. Les pouvoirs locaux à Birnin Lalle. Etudes et travaux du LASDEL 16.

Mohamadou, A. 2004. Foncier agropastoral, conflits et gestion des ressources naturelles au Niger : cas de Dakoro et Abalak. Etudes et Travaux du LASDEL 26.

Olivier de Sardan, J-P. 1998. Chefs et projets au village. Le bulletin de l'APAD 15, 65-89 : « Les dimensions sociales et économiques du développement local et la décentralisation en Afrique au Sud du Sahara » (eds) Nassirou Bako-Arifari \& Pierre-Joseph Laurent.

Olivier de Sardan, J-P \& M. Tijani Alou (eds) A paraître. Introduction : Pouvoirs locaux, espace public et politiques publiques. Paris : Karthala.

Oxby, C. 1989. Les allégeances d'une tribu touarègue entre deux confédérations (Kel Ferwan, Dakoro, Niger). Cahiers de l'IREMAM 7-8. « Touaregs et autres sahariens entre plusieurs mondes : Définitions et redéfinitions de soi et d'autres».

Rapport établi par le chef de la subdivision centrale de Maradi sur le recensement des Peuls de cette subdivision. 1953, $1 \mathrm{E}$ 434, 12 pages.

Rey, M.C. 1989. Limite nord des cultures pluviales : occupation et dégradation d'un environnement sahélien marginal (Dakoro, Niger). Mémoire, Université de Lausanne, Institut de géographie.

Vilmin, M. 1947. Journal de bord. Archives de la Préfecture de Dakoro. 


\section{NOTES}

1. Le Niger a connu une première expérience des partis politiques à la veille de l'indépendance mais ils seront interdits juste après la proclamation de celle-ci.

2. Ces recherches ont donné lieu à des publications dans la série Etudes et Travaux du LASDEL : www.lasdel.net Un article sur les pouvoirs locaux à Birnin Lallé est à paraître dans un ouvrage collectif chez Karthala.

3. Il s'est distingue pendant la famine de 2005 par la mobilisation de plusieurs financements au profit des sinistrés.

4. Entretien avec le chef de canton et Sanoussi Jackou, économiste et homme politique du Kornaka.

5. Hamid Algabit, président du RDP-Jamaa, Sanoussi Jackou, président du PNA-Alouma, Cheiffou Amadou, president du RSD-Gaskiya.

6. Parmi lesquels les infrastructures, une position excentrée, les distances entre chefs lieux, l'accord des communautés, etc.

7. Rassemblement social démocratique.

8. Convention démocratique et sociale.

9. Mouvement National pour la Société du Développement.

\section{RÉSUMÉS}

Dans cet article nous montrons que la décentralisation amorcée au Niger depuis le début des années 90 a eu pour conséquence le renforcement des chefferies locales (cantonales, villageoises et de groupements), la confirmation du primat de la sédentarité sur la mobilité et la marginalisation des éleveurs qui n'ont eu d'autres choix que de s'inscrire dans une logique de sédentarisation pour « obtenir » des chefferies et des «territoires » communaux. Cette évolution vers la territorialisation pourrait constituer une menace pour la survie du mode de vie pastoral dans les régions agricoles et agropastorales au Niger.

In this article we show that the decentralisation initiated in Niger since the early 1990s has led to the strengthening of local chieftaincy (of cantons, villages and groupements), to the primacy of sedentary settlement over mobility, and to the marginalisation of pastoralists left with no other choice than to apply settlement logics to 'obtain' chieftaincies and community 'territories'. This development towards territorialisation could threaten the survival of the pastoral mode of life in pastoral and agropastroal zones.

\section{AUTEUR}

\section{ABDOULAYE MOHAMADOU}

Enseignant-chercheur Faculté d'Agronomie, Université Abdou Moumouni de Niamey, chercheur au LASDEL : azawagh@yahoo.fr 